\title{
Campylobacter jejuni bacteremia and Helicobacter pylori in a patient with $X$-linked agammaglobulinemia
}

\author{
T. van den Bruele • P. E. C. Mourad-Baars • \\ E. C. J. Claas $\cdot$ R. N. van der Plas • E. J. Kuijper • \\ R. G. M. Bredius
}

Received: 15 March 2010 /Accepted: 30 May 2010 / Published online: 17 June 2010

(C) The Author(s) 2010. This article is published with open access at Springerlink.com

\begin{abstract}
We describe a 15-year-old patient with X-linked agammaglobulinemia who developed malabsorption and bacteremia due to infection of Helicobacter pylori and Campylobacter jejuni. The Campylobacter bacteremia was only recognized after subculturing of blood culture bottles that failed to signal in the automated system. After 2 weeks of treatment with meropenem and erythromycin for 4 weeks, the patient developed a relapse of bacteremia 10 months later with a high level erythromycin resistant $C$. jejuni. Sequencing revealed an A2058C mutation in the 23 $\mathrm{S}$ rRNA gene associated with this resistance. Treatment with doxycycline for 4 weeks finally resulted in complete eradication. This case report illustrates the importance for physicians to use adapted culture methods and adequate prolonged therapy in patients with an immunodeficiency. A summary of published case reports and series of patients with hypogammaglobulinemia or agammaglobulinemia with Campylobacter or Helicobacter bacteremia is given.
\end{abstract}

\footnotetext{
Electronic supplementary material The online version of this article (doi:10.1007/s10096-010-0999-7) contains supplementary material, which is available to authorized users.
}

T. van den Bruele · P. E. C. Mourad-Baars · R. N. van der Plas • R. G. M. Bredius $(\bowtie)$

Department of Pediatrics, Leiden University Medical Center, P.O. Box 9600, 2300 RC Leiden, The Netherlands

e-mail: R.G.M.Bredius@lumc.nl

E. C. J. Claas • E. J. Kuijper

Department of Medical Microbiology,

Leiden University Medical Center,

P.O. Box 9600, 2300 RC Leiden, The Netherlands

\section{Introduction}

X-linked agammaglobulinemia (XLA) is a primary immunodeficiency caused by a mutation in the Bruton Tyrosine Kinase $(B T K)$-gene [1]. This defect leads to abnormal development of B-lymphocytes and hypogammaglobulinemia. Patients with XLA usually present with recurrent bacterial infections, mainly of the respiratory and gastrointestinal tract. Other bacterial infections, like cellulitis, arthritis, meningitis and sepsis have also been described, but are less common. Various case reports mention a specific association of XLA patients and patients with acquired hypogammaglobulinemia with bacteremia caused by Helicobacter and Campylobacter species (Table 1) [2, 3].

Recently, we treated a boy with XLA who developed fever, loss of appetite and failure to thrive caused by a combination of $H$. pylori gastritis and recurrent $C$. jejuni bacteremia.

\section{Case report}

A 5-year-old Iranian boy with a history of recurrent infections and an absence of peripheral blood Blymphocytes was diagnosed with XLA. The boy was living in The Netherlands since his fifth year. DNA analysis revealed a frame shift mutation (1614de1T) in exon 16 of the BTK-gene [4]. He was treated with prophylactic cotrimoxazole and intravenous immunoglobulin (IVIG) substitution therapy every 4 weeks. During the last 3 years, $\mathrm{IgG}$ levels varied from 5.0 to $8.7 \mathrm{~g} / \mathrm{L}$ (normal range for $\mathrm{IgG}$ is $5.2-15.0 \mathrm{~g} / \mathrm{L}$ ). To reach a trough level of around $8 \mathrm{~g} / \mathrm{L}$ he required a high dosage of IVIG of $1.2 \mathrm{~g} / \mathrm{kg}$ [5]. He had no detectable IgA and IgM. At the age of 10 years he 


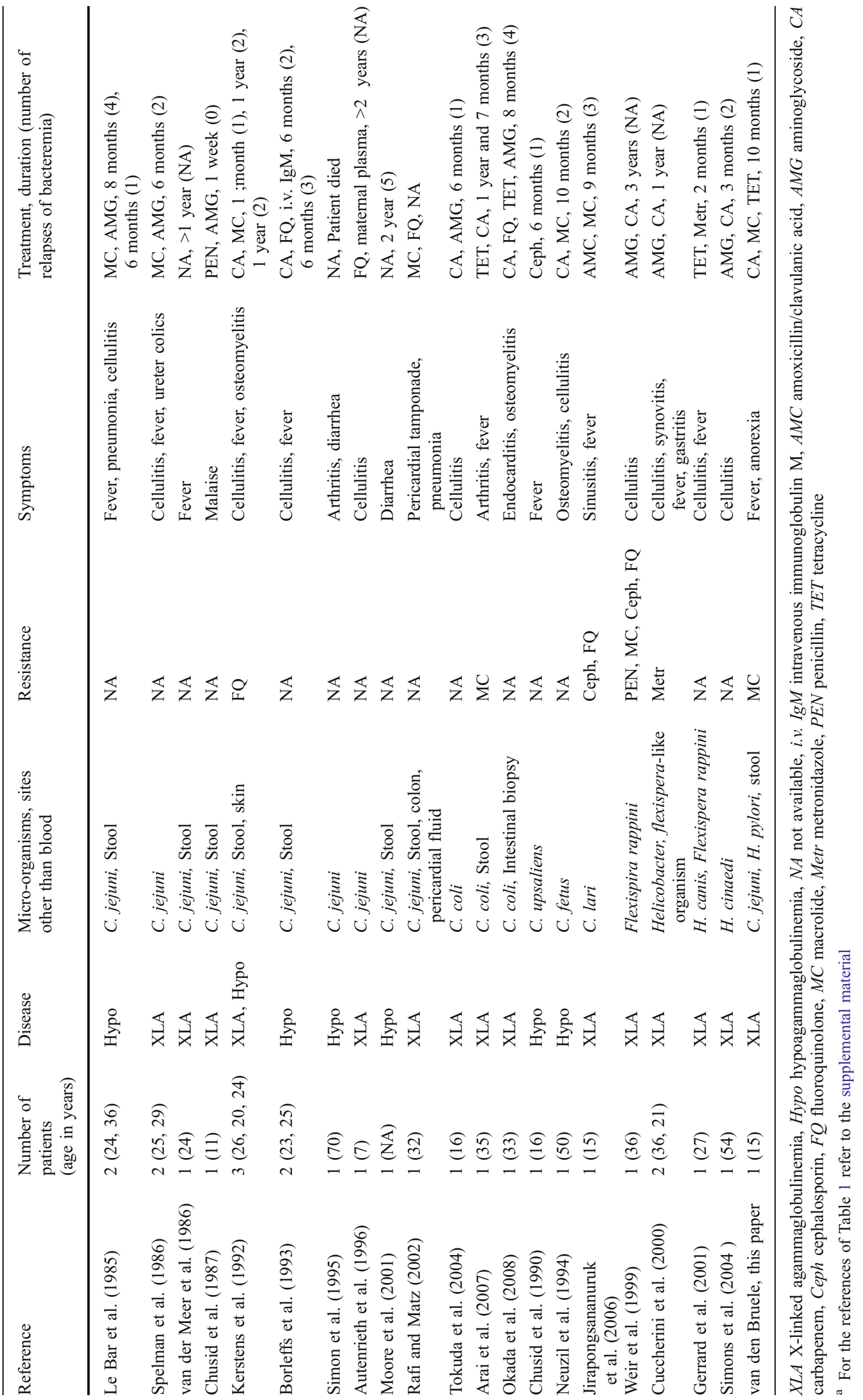


developed epilepsy caused by left occipital lesions, presumably acquired by infections in the past.

At the age of 15 years he was admitted to our hospital with progressive loss of appetite, initially ascribed to his anti-epileptic medication. He was severely malnourished (weight/length ratio -3 Dutch standard deviation score, comparable to Iranian growth charts). Stool antigen test for H. pylori was positive. Endoscopy and biopsies of the gastric antrum, corpus and duodenum showed chronic active inflammation with atrophy and intestinal metaplasia. Cultures of the antrum and corpus were positive for $H$. pylori as well as for C. jejuni. Biopsies of anthrum and corpus were inoculated on non-selective blood agar media and onto specific H. pylori media (Pylori agar, Biomérieux, France) in a micro-aerophilic environment for 7 days. Identification was performed by conventional biochemical reactions according to standard operation procedures [6]. During hospitalization the patient had periods of fever. Laboratory tests showed an elevated C-reactive protein (up to $94 \mathrm{U} / \mathrm{L}$; normal $<5 \mathrm{U} / \mathrm{L}$ ). His leukocyte and neutrophil counts were always normal. Blood cultures revealed $C$. jejuni, sensitive for ciprofloxacin (MIC $0.032 \mathrm{mg} / \mathrm{L}$ ), erythromycin (MIC $0.75 \mathrm{mg} / \mathrm{L}$ ) and meropenem (MIC $0.012 \mathrm{mg} / \mathrm{L}$ ) as determined by $E$-testing (AB Biomérieux, Solna, Sweden). An identical strain was isolated from the patient's feces. Other pathogens were never detected from stool, throat, urine or spinal fluid; cultures or PCR tests were negative for enteric parasites, viruses including enteroviruses and pathogenic bacteria. The patient was treated with intravenous meropenem for 2 weeks, followed by oral erythromycin for another 4 weeks. Repeated blood cultures after 10 days were negative. For eradication of $H$. pylori he additionally received triple therapy with amoxicillin, clarithromycin and omeprazol for one week. H. pylori was sensitive to erythromycin (MIC $0.38 \mathrm{mg} / \mathrm{L}$ ), clarithromycin (MIC $0.19 \mathrm{mg} / \mathrm{L}$ ) and meropenem (MIC $0.002 \mathrm{mg} / \mathrm{L}$ ).

During follow-up he improved clinically and with tube feeding his weight increased to -1 standard deviation score. Endoscopy was repeated 8 months later and showed much less inflammation of the gastric mucosa and absence of $H$. pylori in microscopy and culture of biopsies. However, from a fecal specimen a $C$. jejuni strain was isolated with secondary resistance to erythromycin (MIC $\geq 256 \mathrm{mg} / \mathrm{L}$ ) but persistent sensitivity to ciprofloxacin (MIC $0.125 \mathrm{mg} / \mathrm{L}$ ) and meropenem (MIC $0.008 \mathrm{mg} / \mathrm{L}$ ). Ten months later, he again developed a $C$. jejuni bacteremia. The strain had a similar pattern as the stool isolate, with high level resistance to erythromycin by $E$-testing ( $\mathrm{MIC} \geq 256 \mathrm{mg} / \mathrm{L}$ ), but the strain was sensitive to tetracycline. Sequence analysis of part of the 23S rRNA gene revealed an A2058C mutation (position of nucleotide reported as the Escherichia coli equivalent), known to be associated with erythromycin resistance $[7,8]$. The strain isolated 8 weeks earlier from the feces did not have this mutation. No other pathogens were isolated. He again had complaints of loss of appetite, no diarrhea or skin lesions and some weight loss. Cotrimoxazole prophylactic therapy was switched to azithromycin (for 2 weeks) and later to doxycycline (for 4 weeks), which resulted in clinical improvement and negative stool and blood cultures at follow-up after 6 months.

\section{Discussion}

In the primary antibody deficiency disorders such as XLA, enteropathogenic Helicobacter species and the closely related species Flexispira and Campylobacter can cause persistent gastrointestinal infections and bacteremia, as well as skin and bone infections [2, 3, 9, 10]. In immunocompetent individuals bacteria are eliminated from the blood by antibody- and complement-mediated lysis. Patients with XLA form no or inadequate specific antibodies [1, 11]. Despite immunoglobulin substitution therapy with normal IgG levels (IgA and IgM antibodies remained absent), severe infections with Campylobacter and Helicobacter can emerge [11]. Higher IgG through levels during immunoglobulin supplementation may lead to better protection [5].

In the Netherlands the incidence of Campylobacter infections is 39 per 100,000 people per year (children and adults) [12]. C. jejuni is the most frequently isolated pathogen of the Campylobacter species; C. coli, C. lari and $C$. upsaliensis are less frequently encountered pathogens. Transmission of $C$. jejuni occurs by ingestion of contaminated food (i.e. chicken and pork), water and unpasteurized milk. Helicobacter infections in children are acquired early in life, mainly via oral-fecal contact. In the Netherlands, the prevalence of $H$. pylori in children is very low (1.2\%); most infected children are offspring of nonDutch parents like our patient [13]. Our patient had a double infection, which may be coincidental but could also be based on his Iranian background. In Iran, the prevalence of $H$. pylori was reported to be $58 \%$ in healthy 15 -year-old children [14].

Bacteremia with Campylobacter and Helicobacter species is frequently found in XLA patients. Winkelstein et al. [2] found a prevalence of $10 \%$ in 21 septicemic patients from a series of 201 XLA patients. Recognition of bacteremia with Helicobacter and Campylobacter species is sometimes difficult, since Helicobacter and Campylobacter grow slowly in standard automatic blood culture systems. It is recommended to subculture blood culture bottles to solid media after one week of incubation when no signal of growth has appeared. In some microbiological laboratories this is a standard procedure for blood culture bottles from patients with endocarditis or from patients with hypogammaglobulinemia. In our patient the standard blood 
cultures were negative; only subculturing resulted in recognition of $C$. jejuni.

Symptoms, antibiotic treatment, antibiotic resistance, and duration of bacteremia of published cases are summarized in Table 1. Twenty-six immunocompromised patients with bacteremia caused by Campylobacter or Helicobacter species have been described in the literature; only five of these cases were children ( $<18$ years of age). Remarkably, diarrhea or other gastrointestinal complaints are not always present (33-37\%) [9]. Erysipelas-like cellulitis was present in 11 of 26 patients $(40 \%)$; it mostly disappears within several days after start of antibiotic therapy but is an important early clinical sign for Helicobacter or Campylobacter bacteremia. Five of 25 patients $(20 \%)$ had septic arthritis or osteomyelitis, which is remarkably high; others described an incidence of, respectively, 7\% and 3\% in 201 patients with XLA [2].

Most patients needed prolonged antibiotic therapy to eradicate the microorganisms, with a mean duration of approximately 10 months (ranging from 1 week to 3 years). Our patient presented with fever, malaise and anorexia. He had a long lasting and recurrent bacteremia, which almost exclusively appears in immunocompromised patients.

In immunocompetent individuals, $H$. pylori remains a superficial chronic gastritis, with a small percentage of individuals progressing to duodenal or gastric ulcers and rarely to gastrointestinal malignancies. Common variable immunodeficiency patients have an almost 50-fold increased risk for gastric cancer and a 30 -fold increased risk for lymphoma [11]. The patient we described did not develop a relapse or recurrent $H$. pylori infection.

Antibiotic resistance of Campylobacter species to fluoroquinolones and macrolides is increasing. In The Netherlands, resistance to fluoroquinolones and macrolides is up to $35 \%$ and $2.7 \%$ of the isolates, respectively [12]. However, the latter may be overestimated, since phenotypical routine susceptibility tests for erythromycin are difficult to interpret [15]. Most Campylobacter isolates are sensitive for amoxicillin-clavulanic acid (resistance rate 1-2\%) [9]. The strain of our patient had high-level resistance to erythromycin (MIC $\geq 256 \mathrm{mg} / \mathrm{L}$ ). Because of reported difficulties in the phenotypic recognition of erythromycin resistant isolates, the presence of the erythromycin resistant C. jejuni was confirmed by $23 \mathrm{~S}$ rRNA gene sequence; analysis revealed an A2058C mutation [7, 15]. Campylobacter isolates displaying resistance to erythromycin usually have resistance-associated mutations at position 2058 and/or 2059 of the 23S rRNA gene. They lead to crossresistance to macrolide and lincosamide antibiotics. No resistance has been described for carbapenem antibiotics, such as meropenem [9].

The mortality of inadequately treated bacteremia is estimated to be around $2.5-5.5 \%$ [9]. The mean duration of treatment of bacteremia is 10 to 14 days in immunocompetent individuals. In immunocompromised patients longer treatment may be necessary. Also, in our patient the Campylobacter was hard to eradicate. He received 2 weeks intravenous treatment with meropenem, followed by 4 weeks erythromycin with apparently good effect. However, despite changes of antibiotic treatment $C$. jejuni remained detectable in his stool cultures, and later on he had a relapse of bacteremia. After a 4-week course of doxycycline, $C$. jejuni could be eradicated.

In conclusion, multiple infections can be encountered in patients with XLA that present with vague symptoms such as failure to thrive, weight loss or anorexia and fever. Bacteremia with the Campylobacter species is hard to detect and needs specific laboratory expertise. In patients with agammaglobulinemia, Campylobacter eradication can only be achieved with adequate antibiotic therapy and prolonged treatment; H. pylori seems easier to eliminate.

Acknowledgement The authors wish to thank Ms. A. de Hullu for nutritional support of the patient throughout the disease course.

Open Access This article is distributed under the terms of the Creative Commons Attribution Noncommercial License which permits any noncommercial use, distribution, and reproduction in any medium, provided the original author(s) and source are credited.

\section{References}

1. Conley ME, Broides A, Hernandez-Trujillo V, Howard V, Kanegane H, Miyawaki T, Shurtleff SA (2005) Genetic analysis of patients with defects in early B-cell development. Immunol Rev 203:216-234

2. Winkelstein JA, Marino MC, Lederman HM, Jones SM, Sullivan $\mathrm{K}$, Burks AW, Conley ME, Cunningham-Rundles C, Ochs HD (2006) X-linked agammaglobulinemia: report on a United States registry of 201 patients. Medicine (Baltimore) 85:193-202

3. Freeman AF, Holland SM (2007) Persistent bacterial infections and primary immune disorders. Curr Opin Microbiol 10:70-75

4. Jansen AG, Noordzij JG, Brocker-Vriends AH, van Dongen JJ, van Tol MJ, Bredius RG (2004) Widely divergent clinical phenotype of X-linked agammaglobulinemia in two cousins. Ned Tijdschr Geneeskd 148:1053-1056

5. Eijkhout HW, van der Meer JW, Kallenberg CG, Weening RS, van Dissel JT, Sanders LA, Strengers PF, Nienhuis H, Schellekens PT (2001) The effect of two different dosages of intravenous immunoglobulin on the incidence of recurrent infections in patients with primary hypogammaglobulinemia. A randomized, double-blind, multicenter crossover trial. Ann Intern Med 135:165-174

6. Murray PR, Baron EJ, Jorgensen JH, Landry ML, Pfaller MA (2007) Manual of clinical microbiology. ASM Press, Washington

7. Kuijper EJ, Stevens S, Imamura T, De Wever B, Claas EC (2003) Genotypic identification of erythromycin-resistant campylobacter isolates as helicobacter species and analysis of resistance mechanism. J Clin Microbiol 41:3732-3736

8. Ladely SR, Meinersmann RJ, Englen MD, Fedorka-Cray PJ, Harrison MA (2009) 23S rRNA gene mutations contributing to macrolide resistance in Campylobacter jejuni and Campylobacter coli. Foodborne Pathog Dis 6:91-98 
9. Pacanowski J, Lalande V, Lacombe K, Boudraa C, Lesprit P, Legrand P, Trystram D, Kassis N, Arlet G, Mainardi JL, DoucetPopulaire F, Girard PM, Meynard JL (2008) Campylobacter bacteremia: clinical features and factors associated with fatal outcome. Clin Infect Dis 47:790-796

10. Tokuda K, Nishi J, Miyanohara H, Sarantuya J, Iwashita M, Kamenosono A, Hizukuri K, Wakimoto N, Yoshinaga M (2004) Relapsing cellulitis associated with Campylobacter coli bacteremia in an agammaglobulinemic patient. Pediatr Infect Dis J 23:577-579

11. Desar IM, van Deuren M, Sprong T, Jansen JB, Namavar F, Vandenbroucke-Grauls CM, van der Meer JW (2009) Serum bactericidal activity against Helicobacter pylori in patients with hypogammaglobulinaemia. Clin Exp Immunol 156:434439
12. van Hees BC, Veldman-Ariesen MJ, de Jongh BM, Tersmette M, van Pelt W (2007) Regional and seasonal differences in incidence and antibiotic resistance of Campylobacter from a nationwide surveillance study in The Netherlands: an overview of 20002004. Clin Microbiol Infect 13:305-310

13. Mourad-Baars PE, Verspaget HW, Mertens BJ, Mearin ML (2007) Low prevalence of Helicobacter pylori infection in young children in the Netherlands. Eur J Gastroenterol Hepatol 19:213-216

14. Alborzi A, Soltani J, Pourabbas B, Oboodi B, Haghighat M, Hayati M, Rashidi M (2006) Prevalence of Helicobacter pylori infection in children (south of Iran). Diagn Microbiol Infect Dis 54:259-261

15. van der Beek MT, Claas EC, Mevius DJ, van Pelt W, Wagenaar JA, Kuijper EJ (2010) Inaccuracy of routine susceptibility tests for detection of erythromycin resistance of Campylobacter jejuni and Campylobacter coli. Clin Microbiol Infect 16:51-56 